\title{
Engineering Ethics EduCATIOn: More Than a CEAB ReQuirement
}

\author{
Cindy Rottmann ${ }^{l}$, Doug Reeve ${ }^{1,2}$, Robin Sacks $^{1,2}$, and Mike Klassen ${ }^{l}$ \\ ${ }^{l}$ Institute for Leadership Education in Engineering \\ ${ }^{2}$ Department of Chemical Engineering and Applied Chemistry \\ Faculty of Applied Science and Engineering, \\ University of Toronto \\ cindy.rottmann@utoronto.ca, doug.reeve@utoronto.ca, robin.sacks@utoronto.ca, and mike.klassen@utoronto.ca
}

\begin{abstract}
The Canadian Engineering Accreditation Board (CEAB) requires faculties of engineering to incorporate graduate attribute 3.1.10 "ethics and equity" into their curricula. More than the CEAB requirement, engineering educators have an obligation to prepare students for the ethical dilemmas they will inevitably face in their workplaces and their lives. Our analysis of survey data collected during a pilot study of our ethical case studies project examines a gap between students' perceptions about the effectiveness and importance of engineering ethics education. While there was a considerable range in participants' ratings of the effectiveness of previous engineering ethics coursework, they consistently placed a high value on the importance of ethics in engineering education. This finding is significant because it challenges the prevailing assumption that engineering students' disinterest in non-technical education is the primary barrier to effective ethics instruction.
\end{abstract}

Keywords: engineering, ethics education, case studies, student engagement

\section{INTRODUCTION}

Long before the Canadian Engineering Accreditation Board (CEAB) began requiring faculties of engineering to implement graduate attribute 3.1.10 "Ethics and equity" [1], engineers across the country have been encountering ethical dilemmas. Despite the pervasiveness and inescapabilty of these dilemmas, engineering educators have struggled to teach ethics to undergraduate students in a way that meaningfully engages them [2-11]. There are a few reasons for this struggle. First, ethical dilemmas cannot be easily resolved using technical problem solving strategies, thus necessitating an epistemological shift in thinking for many engineering students. Second, ethics is often relegated to the margins of a tightly packed curriculum giving students insufficient time or space to puzzle over ambiguous, open-ended problems. Third, students with limited workplace experience may not always appreciate the practical relevance of ethical decision-making to their work in chemical processing, infrastructure, programming, mining, or mechanical systems design. Finally, the case studies typically used to teach engineering ethics are either fabricated to highlight a particular clause in the professional code of ethics or too distant from the average university student's experience to be pedagogically meaningful.

The purpose of our paper is to support engineering educators by sharing early results from our engineering ethics education project - an initiative aiming to engage students in philosophical analyses of ethical challenges faced by Canadian engineers across the career trajectory.

\section{METHODOLOGY}

Phase one of our project involved conducting 13 career history interviews with professional engineers and engineering students with industry experience. The focal point of these interviews was a critical incident faced by each individual. The interviews were transcribed verbatim, thematically analyzed and converted into deeply contextualized case studies. We masked the identities of participants through individual and organizational pseudonyms, as well as by altering nonessential details of the cases. We sent draft case studies to interviewees for approval and incorporated their feedback into the final narratives.

Once our first two case studies were drafted and approved, we began phase two - pilot testing the case study method in undergraduate engineering classes. We began with a pre-workshop survey and followed this up with a one hour worked example of an engineering ethics case study. We concluded the class with a post-workshop survey.

The pre-workshop survey included questions about demographics, types of engineering classes in which ethics had been addressed, assessment strategies, topics, pedagogical strategies used by the instructor, and a series of Likert-scale questions about the effectiveness, importance and practicality of engineering ethics instruction to date. We also asked questions about the relatability and authenticity of ethical case studies used by engineering instructors and the extent to which students had felt engaged in engineering ethics education.

The workshop component involved reading one of the case studies, discussing it in small groups and analyzing possible responses as a class. In the end, we generated an organizational map, identified the key issues raised by the case, proposed possible actions to be taken by each of the key actors and then constructed arguments and counterarguments for each action. We concluded the workshop by distributing a handout summarizing eight ethical frameworks. 
Once we had completed our collective analysis of the case, we asked students to complete the "post" workshop survey, which paralleled the structure and content of the "pre" survey. The last question invited students to provide us with unstructured feedback about the case content and method of instruction.

68 students from two engineering leadership classes completed the survey. Our sample was representative of the student body in terms of sex and race, with a slight over-representation of civil engineers and upper year students and a considerable over-representation of students interested in engineering leadership. We analyzed the survey data using a combination of descriptive and inferential statistics. Paired samples t-tests were used to compare pre- and post-workshop measures.

\section{RESULTS \& DISCUSSION}

Paired samples t-tests to be reported elsewhere indicate that students experienced our case study workshop as significantly more practical, relatable, authentic and engaging than previous case study exercises used in their engineering education to date.

For this paper, we compare students' perceptions about the effectiveness and importance of engineering ethics education. We begin by sharing descriptive statistics detailing their engineering experience to date. Next, we use inferential statistics to compare their mean effectiveness ratings with their mean ratings about the importance of engineering ethics. Finally, we parse the data by course type, paying specific attention to class size and pedagogical strategies.

\subsection{Current state of affairs in engineering ethics education}

Before asking students to rate their engineering ethics experiences, we invited them to describe these experiences using structured survey questions. We found that students typically encountered ethical issues in courses dealing with leadership (68\%), design (53\%) and communications $(50 \%)$. To a lesser extent, some also reported that their instructors raised ethical issues in technical courses $(29 \%)$, lab work $(29 \%)$ and tutorials $(26 \%)$. The great majority of students reported pass/fail exams as the primary mode of assessment for engineering ethics coursework (71\%), but some also reported alternative assessment strategies such as class participation (31\%), written assignments (29\%) and presentations $(13 \%)$. The top four topics addressed were plagiarism (91\%), safety $(84 \%)$, engineering failures (75\%) and the Professional Engineers of Ontario (PEO) ethical code $(60 \%)$. Fewer students reported that their instructors raised equity/social justice issues (41\%) or introduced them to philosophical theory $(22 \%)$. The three most common pedagogical strategies used by engineering ethics instructors were issuing warnings- "if your bridge fails, people die!" (76\%), reading legislation (57\%), and WHMIS (Workplace Hazardous Materials Information System) training (47\%). Most directly related to our project, $40 \%$ of students reported being exposed to simple case studies, $35 \%$ to complex case studies and $31 \%$ to spontaneous discussion about ethical issues that arose in class. A forced choice question about optimal learning conditions indicated that $65 \%$ of students learned about engineering ethics best when provided with relatable examples, $31 \%$ when provided with personally interesting content and $4 \%$ when taught by a professor who modeled ethical behavior. Finally, when we asked students whether they believed ethical case studies were better analyzed with a neutral mind or by identifying one's biases, $60 \%$ of students indicated the latter.

\subsection{Debunking the myth that engineering students fail to recognize the importance of ethics}

When we spoke to engineering educators, several of them indicated that it was difficult to engage students in ethics instruction because engineering students tended not to view ethics as an important part of their otherwise technical education. We decided to test this assertion through our survey. Figures one and two below illustrate participants' responses to two survey questions:

1) "Based on these experiences (referring to earlier questions about content, pedagogy and assessment used in engineering ethics education), please rate your engineering ethics instruction at the University of Toronto to date."

2) "Ethical decision-making is an important aspect of engineering education."

Both questions were followed by a zero to ten rating scale.

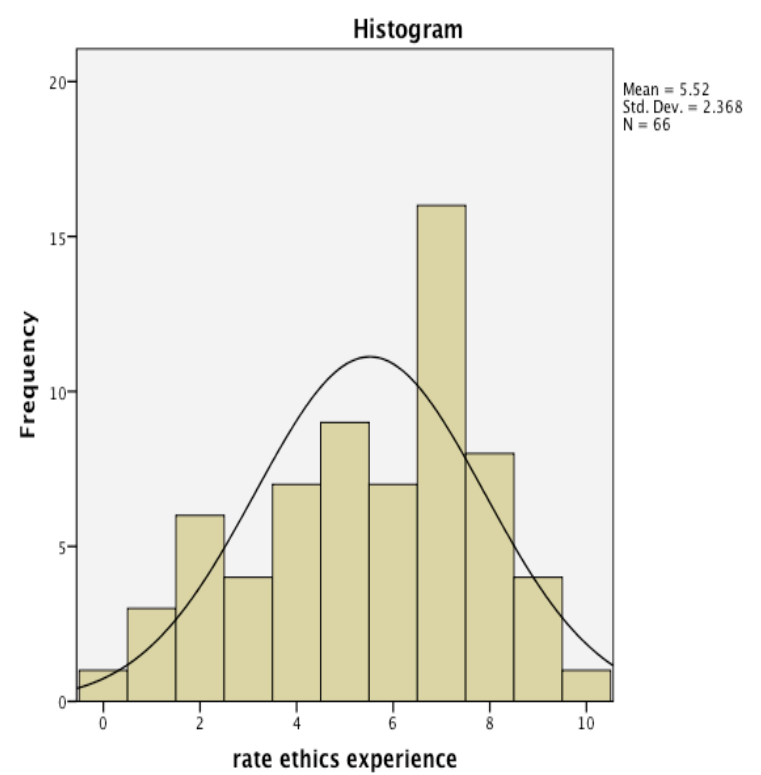

Figure 1: Effectiveness of engineering ethics education 


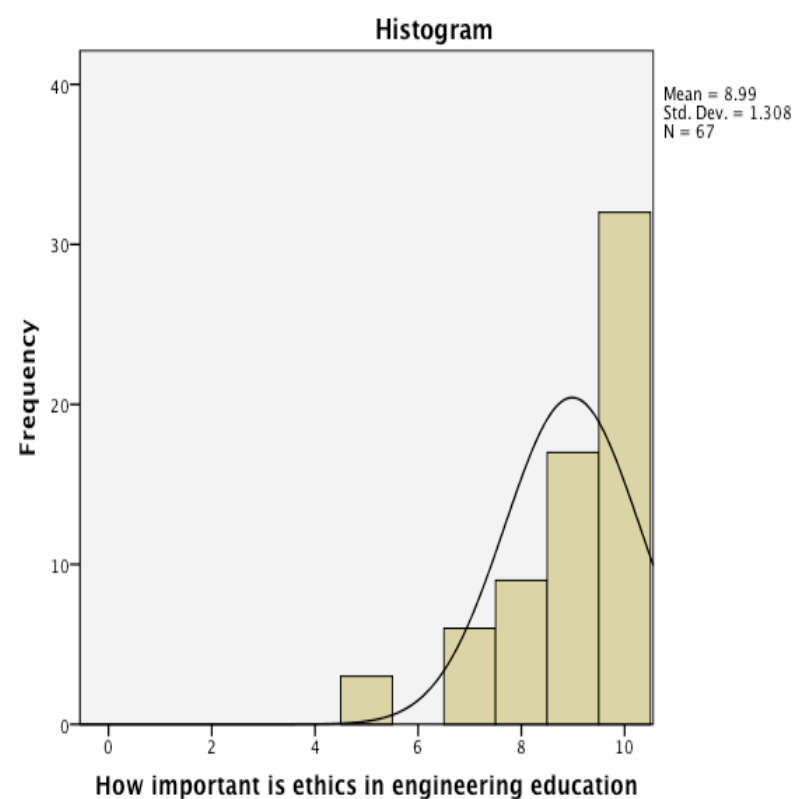

Figure 2: Importance of ethics in engineering education

When we compare the two histograms in figures one and two, it appears that while engineering students have mixed opinions about the effectiveness of engineering ethics instruction to date, they are clear about its importance. The most frequent rating (mode) for this importance question was a ten on a zero to ten-point scale.

We conducted a paired samples t-test to compare engineering students' effectiveness ratings to their importance ratings. The results indicated that the mean importance value $(\mathrm{M}=8.94, \mathrm{SD}=1.32)$ was significantly greater than the mean effectiveness value $(M=5.63$, $\mathrm{SD}=2.29), \mathrm{t}(63)=10.194, \mathrm{p}<.01$. The standard effect size index, d, was 1.27 (very large), with limited overlap in the distributions for the 10 point Likert ratings of prior experience and importance, as shown in figure three below. The $95 \%$ confidence interval for the mean difference between the two ratings was 2.66 to 3.96 .

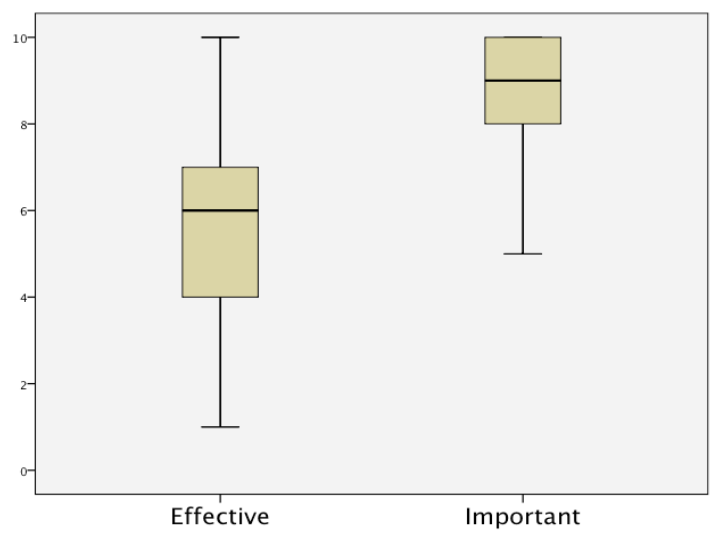

Figure 3: Boxplots of effectiveness and importance ratings

\subsection{Class size and pedagogical strategies}

The considerable variability in effectiveness ratings caused us to parse the data by two types of ethics instruction available to early year engineering students at the University of Toronto: a larger class (1000 students) with a limited range of pedagogical and assessment strategies (lectures, tutorials and exams) and a smaller class (200 students) with a greater diversity of pedagogical and assessment strategies (discussions, readings, case studies, written assignments, class participation, presentations, etc).

$67 \%$ of our sample had taken the larger first year course, $12 \%$ had taken the smaller second year course and $21 \%$ had not taken either. All but two of the students who had selected "none" in the question about ethics education were international exchange students who had taken an ethics primer at the University of Toronto. The two exceptions to this group were domestic students who had transferred from a program with a second year ethics requirement to a program with a first year ethics requirement and had thus missed out on formal ethics instruction. Please see figure 4 below to see how students' effectiveness ratings varied by course type:

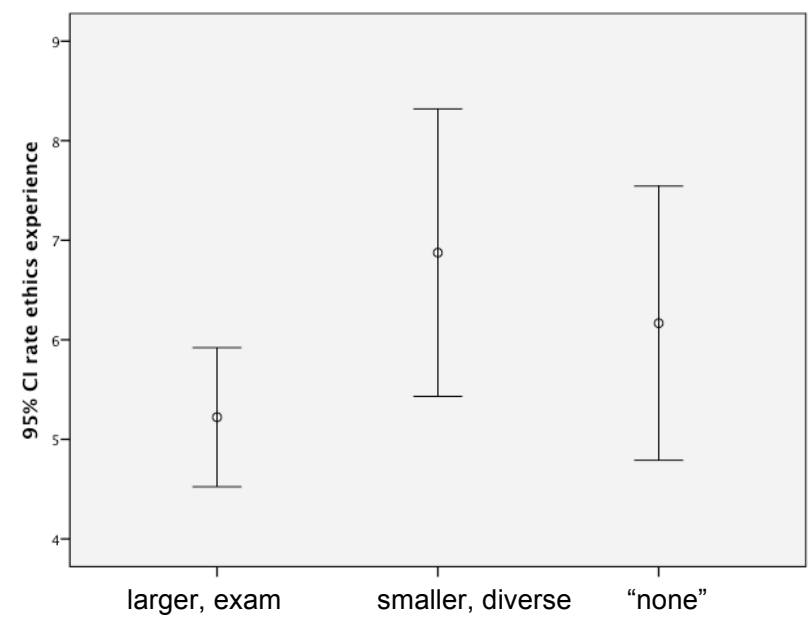

Figure 4: Parsing effectiveness ratings by class size and instructional strategies

Exchange students (those in the "none" category) rated the effectiveness of either their home university's ethics program or the ethics primer they had received at the University of Toronto. Our limited knowledge about the type of ethics instruction these students had received to date caused us to exclude their effectiveness ratings from our inferential statistical analysis.

We conducted an independent samples t-test to evaluate the hypothesis that students enrolled in second year, smaller classes with a range of pedagogical strategies and assessment tools differently rate the quality of their engineering ethics education than students taking first 
year, larger classes with a limited range of assessment tools (pass/fail exam). The test was not significant, $\mathrm{t}(51)=$ $1.9, \mathrm{p}=.06$, however students in smaller classes who had been exposed to a wider diversity of pedagogical and assessment strategies $(\mathrm{M}=6.9, \mathrm{SD}=1.7)$ on average rated the importance of ethics education more highly than students in large classes who had been exposed to a narrower range of pedagogical and assessment strategies $(\mathrm{M}=5.2, \mathrm{SD}=2.3)$. While the mean differences are relatively large, the small number of individuals in our sample in the "smaller/diverse" class $(\mathrm{N}=8)$ decreased the power of the test. The $95 \%$ confidence interval for the difference in means was quite wide, ranging from -.08 to 3.39. The eta square index indicated that $6.7 \%$ of the variance of the rating variable was accounted for by type of ethics coursework (medium effect size).

\section{CONCLUSIONS}

Our pilot study of engineering ethics education suggests that while engineering students have a wide range of views about the effectiveness of their formal ethics instruction, a significant proportion of them view ethics as an important aspect of their engineering education. It is possible that students in our sample - those who elected to enrol in an engineering leadership elective-are more interested in ethical discussions than the full population of undergraduate engineering students at the University of Toronto. Methodological limitations aside, it is important that we not accept presumed disinterest in ethics as a truth in our student body. Rather, we must find ways to engage the next generation of professional engineers in a deep examination of engineering ethics and equity.

Early findings from our pilot study provide preliminary evidence that smaller classes taught later in students' undergraduate programs by instructors who use a diverse range of pedagogical and assessment strategies will assist with engagement levels. A forthcoming article based on this study further suggests that complex case studies based on the actual career experiences of Canadian engineers provide instructors with a practical, relatable, authentic and engaging entry-point to engineering ethics education. By helping students grapple with ambiguous, deeply contextualized situations in a facilitated classroom setting, engineering educators can prepare students to thoughtfully respond to the unexpected ethical issues that are sure to arise in their workplaces and their lives.

Engineering ethics is more than a CEAB requirement. It is a vital aspect of professional development that will serve engineering students along with their future employers, clients and society.

\section{Acknowledgements}

This study was conducted with support from the Dean's Engineering Instructional Innovation Program (EIIP) at the Faculty of Applied Science and Engineering,
University of Toronto. We are grateful to the professional engineers and engineering students who participated in career history interviews, workshops and surveys.

\section{References}

[1] CEAB, Accreditation Criteria and Procedures 2008, 2008, Engineers Canada: Ottawa, ON. p. 24.

[2] Garrett, M.F., What do you think? A series of case studies. Leadership and Management in Engineering, 2006. 6(2): p. 1-2.

[3] Grimson, W. The philosophical nature of engineering: A characterisation of engineering using the language and activities of philosophy. in 114th Annual ASEE Conference and Exposition. 2007. Honolulu, HI: American Society for Engineering Education.

[4] Holsapple, M.A., et al., Framing faculty and student discrepancies in engineering ethics education delivery. Journal of Engineering Education, 2012. 101(2): p. 169-186.

[5] Loui, M.C., Ethics and the development of professional identities of engineering students. Journal of Engineering Education, 2005. 94(4): p. 383-390.

[6] McGinn, R.E., "Mind the gaps": An empirical approach to engineering ethics, 1997-2001. Science and Engineering Ethics, 2003. 9(4): p. 517-542.

[7] Roncin, A. Thoughts on engineering ethics education in Canada. in Canadian Engineering Education Association Conference. 2013. Montreal, QC.

[8] Russell, J.S., Engineering professional growth and quality and cultivating the next generation. Leadership and Management in Engineering, 2008. 8(2): p. 57-60.

[9] Sunderland, M.E., Using student engagement to relocate ethics to the core of the engineering curriculum. Science and Engineering Ethics, 2013. 19.

[10] Sunderland, M.E., Taking emotion seriously: Meeting students where they are. Science and Engineering Ethics, 2014. 20(1): p. 183-195.

[11] Yarmus, J.J., Ethics in professional engineering: Leadership through integrity. Leadership and Management in Engineering, 2010. 10(1): p. 1. 